PROCEEDINGS OF THE

AMERICAN MATHEMATICAL SOCIETY

Volume 131, Number 10, Pages 3297-3304

S 0002-9939(03)07145-4

Article electronically published on May 12, 2003

\title{
THE LANGLANDS CLASSIFICATION FOR NON-CONNECTED $p$-ADIC GROUPS II: MULTIPLICITY ONE
}

\author{
DUBRAVKA BAN AND CHRIS JANTZEN \\ (Communicated by Rebecca Herb)
}

\begin{abstract}
For a non-connected reductive $p$-adic group, we prove that the Langlands subrepresentation appears with multiplicity one in the representation parabolically induced from the corresponding Langlands data.
\end{abstract}

\section{INTRODUCTION}

The Langlands classification for a non-connected reductive $p$-adic group $G$ B-J1] gives a bijective correspondence

$$
\operatorname{Irr}(G) \longleftrightarrow \operatorname{Lang}(G)
$$

between irreducible, admissible representations of $G$ and triples of Langlands data. Let $(P, \nu, \tau)$ be a set of Langlands data (see Definition 2.1) and suppose that $\pi$ is the irreducible representation of $G$ corresponding to $(P, \nu, \tau)$,

$$
\pi \longleftrightarrow(P, \nu, \tau) .
$$

Then $\pi$ is the unique irreducible subrepresentation of the induced representation $i_{G, M}(\exp \nu \otimes \tau)$. This paper proves that the multiplicity of $\pi$ in $i_{G, M}(\exp \nu \otimes \tau)$ is one (cf. Theorem 3.4). Our motivation goes beyond a general interest in extending some useful properties of the Langlands classification to the non-connected case; we have need of them in other work (cf. [B-J2, [J]).

Before closing the introduction, we would like to take the opportunity to thank M. Tadić for conversations helpful to this work.

\section{The LANGlandS Classification}

We take a moment to review the Langlands classification in the context of nonconnected $p$-adic groups (cf. [B-J1]; also see [B-W] and $[\mathrm{S}$ ] for connected $p$-adic groups, $[\mathrm{L}$ for connected real groups, and $[\mathrm{M}]$ for non-connected real groups).

Let $F$ be a $p$-adic field and $G$ the group of $F$-points of a quasi-split reductive algebraic group defined over $F$. Let $G^{0}$ denote the connected component of the identity in $G$. Assume that

$$
C=G / G^{0}
$$

is a finite abelian group.

Received by the editors May 16, 2002.

2000 Mathematics Subject Classification. Primary 22E50.

(C)2003 American Mathematical Society 
We call an irreducible representation of $G$ tempered if its restriction to $G^{0}$ is tempered (cf. Definition 2.5 in B-J1]).

In the group $G^{0}$, fix a Borel subgroup $P_{\emptyset} \subset G^{0}$ and a maximal split torus $A_{\emptyset} \subset P_{\emptyset}$. We let $\Pi$ denote the corresponding set of simple roots. We can choose a set of representatives for $C$ which stabilizes $P_{\emptyset}$, hence acts on $\Pi$. By abuse of notation, we use $C$ for both the component group and this set of representatives.

For $\Phi \subset \Pi$, we let $P_{\Phi}=M_{\Phi} U_{\Phi}$ denote the standard parabolic subgroup of $G^{0}$ determined by $\Phi$. Fix an order on $\Pi$. Then, there is a lexicographic order on subsets of $\Pi$. We define

$$
X_{C}=\left\{\Phi \subset \Pi \mid \Phi \text { is maximal among }\{c \cdot \Phi\}_{c \in C}\right\} .
$$

Let $C(\Phi)=\{c \in C \mid c \cdot \Phi=\Phi\}$ and

$$
M_{\Phi, C(\Phi)}=\left\langle M_{\Phi}, C(\Phi)\right\rangle .
$$

We call $P=M U_{\Phi}$, where $M_{\Phi} \leq M \leq M_{\Phi, C(\Phi)}$ and $\Phi \in X_{C}$, a standard parabolic subgroup of $G$.

Suppose $P$ is a standard parabolic subgroup of $G$. Write $P^{0}=P_{\Phi}$. Let $A$ be the split component of $M_{\Phi}, \mathfrak{a}$ the real Lie algebra of $A$, and $\mathfrak{a}^{*}$ its dual. Let $\Pi\left(P^{0}, A\right) \subset \mathfrak{a}^{*}$ denote the set of simple roots corresponding to the pair $\left(P^{0}, A\right)$. We set

$$
\begin{aligned}
& \mathfrak{a}_{-}^{*}=\left\{x \in \mathfrak{a}^{*} \mid\langle x, \alpha\rangle<0, \forall \alpha \in \Pi\left(P^{0}, A\right)\right\}, \\
& \mathfrak{a}_{-}^{*}(C)=\left\{x \in \mathfrak{a}_{-}^{*} \mid x \succeq c \cdot x, \forall c \in C(\Phi)\right\},
\end{aligned}
$$

where $\langle\cdot, \cdot\rangle$ is a $C(\Phi)$-invariant inner product on $\mathfrak{a}^{*} \times \mathfrak{a}^{*}$ and $\succeq$ is the lexicographic order inherited from the order on $\Pi$ (cf. section 3 of [B-J1] for details).

Definition 2.1 ([B-J1], Definition 4.1). A set of Langlands data for $G$ is a triple $(P, \nu, \tau)$ with the following properties:

1. $P=M U$ is a standard parabolic subgroup of $G$.

2. $\nu \in \mathfrak{a}_{-}^{*}(C)$.

3. $M=M_{\Phi, C(\Phi, \nu)}$, where $C(\Phi, \nu)=\{c \in C(\Phi) \mid c \cdot \nu=\nu\}$.

4. $\tau \in \operatorname{Irr}(M)$ is tempered.

For $\nu \in \mathfrak{a}_{-}^{*}(C)$, let $\exp \nu$ be the character of $M_{\Phi}$ defined by $\exp \nu=q^{\left\langle\nu, H_{\Phi}(\cdot)\right\rangle}$, where $H_{\Phi}: M_{\Phi} \rightarrow \mathfrak{a}$ is the homomorphism defined in [H]. If $\tau$ is a representation of $M$, then $\exp \nu \otimes \tau$ is the representation of $M$ defined by $(\exp \nu \otimes \tau)(m c)=$ $\exp \nu(m) \tau(m c)$ ([B-J1], section 2). As in [B-Z], we let $i_{G, M}(\tau)$ denote the representation of $G$ parabolically induced from $\tau$.

Theorem 2.2 (Langlands classification, B-J1, Theorem 4.2). There is a bijective correspondence

$$
\operatorname{Lang}(G) \longleftrightarrow \operatorname{Irr}(G),
$$

where $\operatorname{Lang}(G)$ denotes the set of all triples of Langlands data. Furthermore, if $(P, \nu, \tau) \leftrightarrow \pi$ under this correspondence, then $\pi$ is the unique irreducible subrepresentation of $i_{G, M}(\exp \nu \otimes \tau)$.

If $(P, \nu, \tau) \leftrightarrow \pi$, then we call $\pi$ the Langlands subrepresentation corresponding to $(P, \nu, \tau)$. 


\section{Multiplicity ONE}

We now take up the proof of multiplicity one for the Langlands classification.

In what follows, it will occasionally be convenient to work in the Grothendieck group setting. Recall that in this context, we write $\pi_{1} \leq \pi_{2}$ if $m\left(\tau, \pi_{1}\right) \leq m\left(\tau, \pi_{2}\right)$ for every smooth, irreducible representation $\tau$ of $G$, where $m(\tau, \pi)=$ multiplicity of $\tau$ in $\pi$.

Let $\mathfrak{a}_{0}$ denote the real Lie algebra of $A_{\emptyset}$ and $\mathfrak{a}_{0}^{*}$ its dual. Recall that for a standard parabolic subgroup, we may identify $\mathfrak{a}^{*}$ as a subspace of $\mathfrak{a}_{0}^{*}$. Let $>$ be the order from section XI.2 in $\mathrm{B}-\mathrm{W}$, i.e.,

$$
\mu>\nu \text { if }\langle\mu-\nu, \alpha\rangle>0, \text { for all } \alpha \in \Pi,
$$

or equivalently, $\mu-\nu \in\left(\mathfrak{a}_{0}^{*}\right)_{+}$. We write $\mu \geq \nu$ if $\langle\mu-\nu, \alpha\rangle \geq 0$, for all $\alpha \in \Pi$. Note that $\mu \geq \nu$ and $\nu \geq \mu$ imply that $\mu-\nu$ is in the center of $\mathfrak{a}_{0}^{*}$ (e.g., see [B-W], XI.1).

Lemma 3.1. Let $\mu, \nu \in \mathfrak{a}_{0}^{*}$.

1. If $\mu>\nu$, then $c \cdot \mu>c \cdot \nu$, for all $c \in C$.

2. For all $c \in C, \mu \ngtr c \cdot \mu$ and $\mu \nless c \cdot \mu$.

Proof. 1. This follows immediately from the fact that $c \cdot \Pi=\Pi$ and the fact that the inner product on $\mathfrak{a}_{0}^{*} \times \mathfrak{a}_{0}^{*}$ may be taken to be $C$-invariant (cf. section 2 in B-J1]).

2. Suppose $\mu>c \cdot \mu$, for some $c \in C$. Let $m$ be the order of $c$. Then, according to assertion $1, \mu>c \cdot \mu$ implies $c \cdot \mu>c^{2} \cdot \mu, c^{2} \cdot \mu>c^{3} \cdot \mu$, etc. Thus,

$$
\mu>c \cdot \mu>c^{2} \cdot \mu>\cdots>c^{m} \cdot \mu=\mu,
$$

a contradiction.

We now define an ordering on the $C$-orbits in $\mathfrak{a}_{0}^{*}$ and show it is well defined.

Definition 3.2. Let $\mu, \nu \in \mathfrak{a}_{0}^{*}$. We write $C \cdot \mu>C \cdot \nu$ if $c_{1} \cdot \mu>c_{2} \cdot \nu$, for some $c_{1}, c_{2} \in C$.

Lemma 3.3. The ordering in Definition 3.2 is well defined. In particular, suppose $\mu, \nu \in \mathfrak{a}_{0}^{*}$. Then, the following hold:

1. If $C \cdot \mu>C \cdot \nu$, then $C \cdot \nu \ngtr C \cdot \mu$.

2. $C \cdot \mu \ngtr C \cdot \mu$.

Proof. 1. Suppose $C \cdot \mu>C \cdot \nu$ and $C \cdot \nu>C \cdot \mu$. According to Lemma 3.1, there exist $c_{1}, c_{2} \in C$ such that $\mu>c_{1} \cdot \nu$ and $\nu>c_{2} \cdot \mu$. Then, by Lemma 3.1, 1,

$$
\mu>c_{1} \cdot \nu>c_{1} c_{2} \cdot \mu
$$

which is a contradiction (Lemma $3.1,2$ ).

2. Follows from Lemma 3.1, 2.

Theorem 3.4. Let $\pi$ be an irreducible representation of $G$ having Langlands data $(P, \nu, \tau)$. Then,

1. $\pi$ appears with multiplicity one in $i_{G, M}(\exp \nu \otimes \tau)$.

2. Suppose that $\theta$ is an irreducible component of $i_{G, M}(\exp \nu \otimes \tau)$ with Langlands data $\left(P_{\theta}, \nu_{\theta}, \tau_{\theta}\right)$. Then, $C \cdot \nu<C \cdot \nu_{\theta}$ or $\nu=\nu_{\theta}$. Furthermore, equality occurs if and only if $\theta \cong \pi$. 
Proof. Let

$$
G^{0}=G_{0} \subset G_{1} \subset \cdots \subset G_{k}=G,
$$

where $\left|G_{i} / G_{i-1}\right|$ is prime for $i=1, \ldots, k$. Recall that the proof of the Langlands classification in B-J1 follows the lead of G-H], using a result of G-K] (cf. Lemma 2.1 in [B-J1] for a precise formulation) to work inductively up the filtration. Thus, as in [B-J1], we argue inductively, assuming that multiplicity one holds for $G_{i-1}$ and showing that it holds for $G_{i}$. Multiplicity one for the connected group $G^{0}$ is a classical result (e.g., see $[\mathrm{B}-\mathrm{W}]$ ).

For convenience, let $G_{1} \subset G_{2}$ be two consecutive groups in the filtration above (not necessarily the first two). Then $G_{1} / G^{0}=C_{1}$ and $G_{2} / G^{0}=C_{2}$ with $C_{1} \subset$ $C_{2} \subset C$ and $\left|C_{2} / C_{1}\right|$ prime. Write $D=C_{2} / C_{1}$.

Let $\pi_{1}$ be an irreducible representation of $G_{1}$ with Langlands data $\left(P_{1}, \nu_{1}, \tau_{1}\right)$. Write $P_{1}^{0}=P_{\Phi_{1}}$. For $i=1,2$, let

$$
\begin{aligned}
C_{i}\left(\Phi_{1}\right) & =\left\{c \in C_{i} \mid c \cdot \Phi_{1}=\Phi_{1}\right\}, \\
C_{i}\left(\Phi_{1}, \nu_{1}\right) & =\left\{c \in C_{i} \mid c \cdot \Phi_{1}=\Phi_{1} \text { and } c \cdot \nu_{1}=\nu_{1}\right\} \\
C_{i}\left(\Phi_{1}, \nu_{1}, \tau_{1}\right) & =\left\{c \in C_{i} \mid c \cdot \Phi_{1}=\Phi_{1}, c \cdot \nu_{1}=\nu_{1} \text { and } c \cdot \tau_{1}=\tau_{1}\right\} .
\end{aligned}
$$

According to [B-J1, Lemma 4.3, we have either $C_{2}(\cdot)=C_{1}(\cdot)$ or $C_{2}(\cdot) / C_{1}(\cdot) \cong$ $D$.

Lemma 3.5. Assume Theorem 3.4 holds for $G_{1}$. Let $\pi_{2}$ be an irreducible representation of $G_{2}$ having Langlands data $\left(P_{2}, \nu_{2}, \tau_{2}\right)$. Then $\pi_{2}$ appears with multiplicity one in $i_{G_{2}, M_{2}}\left(\exp \nu_{2} \otimes \tau_{2}\right)$.

Proof. Let $r_{G_{1}, G_{2}}\left(\pi_{2}\right)=\left.\pi_{2}\right|_{G_{1}}$. Let $\pi_{1}$ be an irreducible subquotient of $r_{G_{1}, G_{2}}\left(\pi_{2}\right)$. We denote its Langlands data by $\left(P_{1}, \nu_{1}, \tau_{1}\right)$. Recall that the proof of the Langlands classification in [B-J1] considers four cases:

1. $C_{2}\left(\Phi_{1}\right)=C_{1}\left(\Phi_{1}\right)$.

2. $C_{2}\left(\Phi_{1}\right) \neq C_{1}\left(\Phi_{1}\right)$ but $C_{2}\left(\Phi_{1}, \nu_{1}\right)=C_{1}\left(\Phi_{1}, \nu_{1}\right)$.

3. $C_{2}(\Phi) \neq C_{1}(\Phi), C_{2}\left(\Phi, \nu_{1}\right) \neq C_{1}\left(\Phi, \nu_{1}\right)$ but $C_{2}\left(\Phi, \nu_{1}, \tau_{1}\right)=C_{1}\left(\Phi, \nu_{1}, \tau_{1}\right)$.

4. $C_{2}(\Phi) \neq C_{1}(\Phi), C_{2}\left(\Phi, \nu_{1}\right) \neq C_{1}\left(\Phi, \nu_{1}\right), C_{2}\left(\Phi, \nu_{1}, \tau_{1}\right) \neq C_{1}\left(\Phi, \nu_{1}, \tau_{1}\right)$.

We claim the following: If $c \in C_{2}$ and $c \cdot \pi_{1} \not \pi_{1}$, then $c \cdot \pi_{1}$ is not a subquotient of $i_{G_{1}, M_{1}}\left(\exp \nu_{1} \otimes \tau_{1}\right)$. If $\left(P_{1}, \nu_{1}, \tau_{1}\right)$ fits into case 1 above, then $c \cdot \pi_{1}$ has Langlands data $\left(c \cdot P_{1}, c \cdot \nu_{1}, c \cdot \tau_{1}\right)$ (cf. Proposition 4.5 in [B-J1]). However, if $\theta_{1}$ is an irreducible subquotient of $i_{G_{1}, M_{1}}\left(\exp \nu_{1} \otimes \tau_{1}\right)$ having Langlands data $\left(P_{\theta_{1}}, \nu_{\theta_{1}}, \tau_{\theta_{1}}\right)$, then Theorem 3.4 for $G_{1}$ implies $C_{1} \cdot \nu_{\theta_{1}}>C_{1} \cdot \nu_{1}$ or $\nu_{\theta_{1}}=\nu_{1}, \theta_{1} \cong \pi_{1}$. Since this is not the case for $c \cdot \pi_{1}$ (cf. Lemma 3.1,2), it follows that $c \cdot \pi_{1}$ is not a subquotient of $i_{G_{1}, M_{1}}\left(\exp \nu_{1} \otimes \tau_{1}\right)$. The argument when $\left(P_{1}, \nu_{1}, \tau_{1}\right)$ is case 2 is essentially identical. The only remaining possibility is case 3 (case 4 has $c \cdot \pi_{1} \cong \pi_{1}$, for all $c \in C_{2}$ ). We note that in this case $\nu_{c \cdot \pi_{1}}=c \cdot \nu_{1}=\nu_{1}$. Since $\pi_{1}$ is the only irreducible subquotient of $i_{G_{1}, M_{1}}\left(\exp \nu_{1} \otimes \tau_{1}\right)$ having exponent $\nu_{1}$ (Theorem 3.4 for $G_{1}$ ), we see that $c \cdot \pi_{1}$ is not a subquotient of $i_{G_{1}, M_{1}}\left(\exp \nu_{1} \otimes \tau_{1}\right)$, finishing the claim.

We first consider the cases which have $i_{G_{2}, G_{1}}\left(\pi_{1}\right) \cong \pi_{2}$, that is, when $\left(P_{1}, \nu_{1}, \tau_{1}\right)$ is case 1,2 or 3 . In these three cases, we claim that

$$
i_{G_{2}, G_{1}} \circ i_{G_{1}, M_{1}}\left(\exp \nu_{1} \otimes \tau_{1}\right) \cong i_{G_{2}, M_{2}}\left(\exp \nu_{2} \otimes \tau_{2}\right) .
$$


For case 1, recall from [B-J1] that $\left(P_{2}, \nu_{2}, \tau_{2}\right)=\left(c \cdot P_{1}, c \cdot \nu_{1}, c \cdot \tau_{1}\right)$, for some $c \in C_{2}$. Note that $c \cdot P_{1}$ is a standard parabolic subgroup ("not" in the proof of Lemma 4.4 in [B-J1] is a typo; the set of representatives for $C$ stabilizes $P_{\emptyset}$, so $P_{\emptyset} \subseteq c \cdot P_{1}$ ). By Lemma 4.4 in B-J1,

$$
i_{G_{1}, c \cdot M_{1}}\left(\exp c \cdot \nu_{1} \otimes c \cdot \tau_{1}\right) \cong c \cdot i_{G_{1}, M_{1}}\left(\exp \nu_{1} \otimes \tau_{1}\right) .
$$

Therefore,

$$
\begin{aligned}
i_{G_{2}, M_{2}}\left(\exp \nu_{2} \otimes \tau_{2}\right) & \cong i_{G_{2}, G_{1}} \circ i_{G_{1}, c \cdot M_{1}}\left(\exp c \cdot \nu_{1} \otimes c \cdot \tau_{1}\right) \\
& \cong i_{G_{2}, G_{1}} \circ c \circ i_{G_{1}, M_{1}}\left(\exp \nu_{1} \otimes \tau_{1}\right) \\
& \cong i_{G_{2}, G_{1}} \circ i_{G_{1}, M_{1}}\left(\exp \nu_{1} \otimes \tau_{1}\right)
\end{aligned}
$$

as claimed. For case $2,\left(P_{2}, \nu_{2}, \tau_{2}\right)=\left(P_{1}, c \cdot \nu_{1}, c \cdot \tau_{1}\right)$, for some $c \in C_{2}$. The argument is almost identical to that for case 1; we omit the details. For case 3, $\left(P_{2}, \nu_{2}, \tau_{2}\right)=\left(P_{\Phi, C_{2}\left(\Phi, \nu_{1}\right)}, \nu_{1}, i_{M_{\Phi, C_{2}\left(\Phi, \nu_{1}\right)}, M_{1}}\left(\tau_{1}\right)\right)$. In this case,

$$
\begin{aligned}
i_{G_{2}, M_{\Phi, C_{2}\left(\Phi, \nu_{1}\right)}}\left(\exp \nu_{1} \otimes i_{M_{\Phi, C_{2}\left(\Phi, \nu_{1}\right)}, M_{1}}\left(\tau_{1}\right)\right) & \cong i_{G_{2}, M_{1}}\left(\exp \nu_{1} \otimes \tau_{1}\right) \\
& \cong i_{G_{2}, G_{1}} \circ i_{G_{1}, M_{1}}\left(\exp \nu_{1} \otimes \tau_{1}\right),
\end{aligned}
$$

as needed.

To see multiplicity one for cases 1,2 and 3 , write

$$
i_{G_{1}, M_{1}}\left(\exp \nu_{1} \otimes \tau_{1}\right)=\pi_{1}+\sum_{i} \theta_{1}^{(i)}
$$

in the Grothendieck group. From the preceding claim,

$$
i_{G_{2}, M_{2}}\left(\exp \nu_{2} \otimes \tau_{2}\right)=i_{G_{2}, G_{1}}\left(\pi_{1}\right)+\sum_{i} i_{G_{2}, G_{1}}\left(\theta_{1}^{(i)}\right) .
$$

Observe that $i_{G_{2}, G_{1}}\left(\pi_{1}\right) \cong \pi_{2}$. Theorem 3.4 for $G_{1}$ tells us that $\pi_{1} \neq \theta_{1}^{(i)}$. Furthermore, $c \cdot \pi_{1} \not \pi_{1}$ is not a subquotient of $i_{G_{1}, M_{1}}\left(\exp \nu_{1} \otimes \tau_{1}\right)$. Therefore, $\pi_{2}$ appears with multiplicity one in $i_{G_{2}, M_{2}}\left(\exp \nu_{2} \otimes \tau_{2}\right)$.

We now consider the remaining case, that is, when $\left(P_{1}, \nu_{1}, \tau_{1}\right)$ is case 4 . In this case, we have

$$
i_{G_{2}, M_{1}}\left(\exp \nu_{1} \otimes \tau_{1}\right) \cong i_{G_{2}, M_{\Phi, C_{2}\left(\Phi, \nu_{1}\right)}}\left(\exp \nu_{1} \otimes \bigoplus_{\chi \in \hat{D}} \chi \tau_{2}\right) .
$$

Thus, it suffices to show that $\pi_{2}$ appears with multiplicity one in $i_{G_{2}, M_{1}}\left(\exp \nu_{1} \otimes \tau_{1}\right)$. Write

$$
i_{G_{1}, M_{1}}\left(\exp \nu_{1} \otimes \tau_{1}\right)=\pi_{1}+\sum_{i} \theta_{1}^{(i)} .
$$

Then,

$$
i_{G_{2}, M_{2}}\left(\exp \nu_{2} \otimes \tau_{2}\right)=i_{G_{2}, G_{1}}\left(\pi_{1}\right)+\sum_{i} i_{G_{2}, G_{1}}\left(\theta_{1}^{(i)}\right)
$$

in the Grothendieck group. Observe that $i_{G_{2}, G_{1}}\left(\pi_{1}\right)$ contains one copy of $\pi_{2}$, and $\pi_{2}$ is not a subquotient of $i_{G_{2}, G_{1}}\left(\theta_{1}^{(i)}\right.$ ) (by multiplicity one for $G_{1}$ and the fact that if $\theta_{1}$ is irreducible such that $\pi_{2}$ is a subquotient of $i_{G_{2}, G_{1}}\left(\theta_{1}\right)$, then $\left.\theta_{1} \cong \pi_{1}\right)$. Therefore, $\pi_{2}$ appears with multiplicity one in $i_{G_{2}, M_{2}}\left(\exp \nu_{2} \otimes \tau_{2}\right)$, as needed. 
Lemma 3.6. Assume Theorem 3.4 holds for $G_{1}$. Let $\left(P_{1}, \nu_{1}, \tau_{1}\right)$ be a set of Langlands data for $G_{1}$, with corresponding Langlands subrepresentation $\pi_{1}$. Let $\theta_{1}$ be an irreducible subquotient of $i_{G_{1}, M_{1}}\left(\exp \nu_{1} \otimes \tau_{1}\right)$. Suppose

$$
\pi \leq i_{G_{2}, G_{1}}\left(\pi_{1}\right), \quad \theta \leq i_{G_{2}, G_{1}}\left(\theta_{1}\right)
$$

are irreducible subquotients with Langlands data $(P, \nu, \tau)$ and $\left(P_{\theta}, \nu_{\theta}, \tau_{\theta}\right)$, respectively. Then $C_{2} \cdot \nu \leq C_{2} \cdot \nu_{\theta}$. Furthermore, if equality occurs, then $\theta$ is a component of $i_{G_{2}, G_{1}}\left(\pi_{1}\right)$.

Proof. Let $\left(P_{\theta_{1}}, \nu_{\theta_{1}}, \tau_{\theta_{1}}\right)$ be the Langlands data for $\theta_{1}$. From the construction of Langlands data in B-J1, we have $\nu_{\theta}=c_{\theta} \cdot \nu_{\theta_{1}}$ and $\nu=c_{\pi} \cdot \nu_{1}$, for some $c_{\theta}, c_{\pi} \in C_{2}$. Since $\theta_{1}$ is a subquotient of $i_{G_{1}, M_{1}}\left(\exp \nu_{1} \otimes \tau_{1}\right)$, Theorem 3.4 for $G_{1}$ tells us $C_{1} \cdot \nu_{1}<C_{1} \cdot \nu_{\theta_{1}}$ or $\nu_{1}=\nu_{\theta_{1}}$, with equality if and only if $\theta_{1} \cong \pi_{1}$. Therefore, $C_{2} \cdot \nu \leq C_{2} \cdot \nu_{\theta}$, as claimed. Furthermore, if equality occurs, then $\theta \leq i_{G_{2}, G_{1}}\left(\theta_{1}\right) \cong i_{G_{2}, G_{1}}\left(\pi_{1}\right)$.

Lemma 3.7. Assume Theorem 3.4 holds for $G_{1}$. Let $\left(P_{2}, \nu_{2}, \tau_{2}\right)$ be a set of Langlands data for $G_{2}$ and $\pi_{2}$ the corresponding Langlands subrepresentation. Suppose $\theta$ is an irreducible subquotient of $i_{G_{2}, M_{2}}\left(\exp \nu_{2} \otimes \tau_{2}\right)$ having Langlands data $\left(P_{\theta}, \nu_{\theta}, \tau_{\theta}\right)$. Then $C_{2} \cdot \nu_{2}<C_{2} \cdot \nu_{\theta}$ or $\nu_{2}=\nu_{\theta}$. Furthermore, if equality occurs, then $\theta \cong \pi_{2}$.

Proof. Recall from the proof of the Langlands classification in the non-connected case that there are three possibilities: If $P_{1}=P_{2} \cap G_{1}$, we may have

1. $P_{2}=P_{1}$.

2. $P_{2} \neq P_{1}$ and $r_{M_{1}, M_{2}}(\tau)$ is reducible.

3. $P_{2} \neq P_{1}$ and $r_{M_{1}, M_{2}}(\tau)$ is irreducible.

We break the proof into these three cases.

In case $1,\left(P_{2}, \nu_{2}, \tau_{2}\right)=\left(P_{1}, \nu_{1}, \tau_{1}\right)$ is also a set of Langlands data for $G_{1}$; let $\pi_{1}$ denote the corresponding Langlands subrepresentation. Then, $\pi_{2} \cong i_{G_{2}, G_{1}}\left(\pi_{1}\right)$. If $\theta$ is an irreducible subquotient of $i_{G_{2}, M_{2}}\left(\exp \nu_{2} \otimes \tau_{2}\right)$, then

$$
\theta \leq i_{G_{2}, M_{2}}\left(\exp \nu_{2} \otimes \tau_{2}\right) \cong i_{G_{2}, G_{1}} \circ i_{G_{1}, M_{1}}\left(\exp \nu_{1} \otimes \tau_{1}\right),
$$

so $\theta$ is a subquotient of $i_{G_{2}, G_{1}}\left(\theta_{1}\right)$ for some irreducible $\theta_{1} \leq i_{G_{1}, M_{1}}\left(\exp \nu_{1} \otimes \tau_{1}\right)$. Lemma 3.6 implies $C_{2} \cdot \nu_{2} \leq C_{2} \cdot \nu_{\theta}$. Furthermore, if equality occurs, then $\theta \leq$ $i_{G_{2}, G_{1}}\left(\pi_{1}\right) \cong \pi_{2}$, so $\theta \cong \pi_{2}$ and $\nu_{\theta}=\nu_{2}$.

In the second case, let $\nu_{1}=\nu_{2}$ and let $\tau_{1}$ be an irreducible subquotient of $r_{M_{1}, M_{2}}\left(\tau_{2}\right)$. Then $\left(P_{1}, \nu_{1}, \tau_{1}\right)$ is a set of Langlands data for $G_{1}$; let $\pi_{1}$ denote the corresponding Langlands subrepresentation. We have $\pi_{2} \cong i_{G_{2}, G_{1}}\left(\pi_{1}\right)$. If $\theta$ is an irreducible subquotient of $i_{G_{2}, M_{2}}\left(\exp \nu_{2} \otimes \tau_{2}\right)$, then (noting $i_{M_{2}, M_{1}}\left(\tau_{1}\right) \cong \tau_{2}$ )

$$
\begin{aligned}
\theta & \leq i_{G_{2}, M_{2}}\left(\exp \nu_{2} \otimes \tau_{2}\right) \cong i_{G_{2}, M_{2}}\left(\exp \nu_{2} \otimes i_{M_{2}, M_{1}}\left(\tau_{1}\right)\right) \\
& \cong i_{G_{2}, M_{1}}\left(\exp \nu_{1} \otimes \tau_{1}\right) \cong i_{G_{2}, G_{1}} \circ i_{G_{1}, M_{1}}\left(\exp \nu_{1} \otimes \tau_{1}\right),
\end{aligned}
$$

so $\theta$ is a subquotient of $i_{G_{2}, G_{1}}\left(\theta_{1}\right)$ for some irreducible $\theta_{1} \leq i_{G_{1}, M_{1}}\left(\exp \nu_{1} \otimes \tau_{1}\right)$. The result then follows from Lemma 3.6 in the same way as in case 1 .

In case 3 , let $\nu_{1}=\nu_{2}$ and $\tau_{1}=r_{M_{1}, M_{2}}\left(\tau_{2}\right)$. Then $\left(P_{1}, \nu_{1}, \tau_{1}\right)$ is a set of Langlands data for $G_{1}$; let $\pi_{1}$ denote the corresponding Langlands subrepresentation. Then,

$$
i_{G_{2}, G_{1}}\left(\pi_{1}\right)=\bigoplus_{\chi \in \hat{D}} \chi \pi_{2}
$$


Observe that

$$
i_{G_{2}, G_{1}} \circ i_{G_{1}, M_{1}}\left(\exp \nu_{1} \otimes \tau_{1}\right) \cong i_{G_{2}, M_{2}}\left(\exp \nu_{2} \otimes \bigoplus_{\chi \in \hat{D}} \chi \tau_{2}\right) .
$$

Write $i_{G_{1}, M_{1}}\left(\exp \nu_{1} \otimes \tau_{1}\right)=\pi_{1}+\sum_{i} \theta_{1}^{(i)}$ in the Grothendieck group. Then

$$
\begin{aligned}
i_{G_{2}, G_{1}} \circ i_{G_{1}, M_{1}}\left(\exp \nu_{1} \otimes \tau_{1}\right) & =i_{G_{2}, G_{1}}\left(\pi_{1}\right)+\sum_{i} i_{G_{2}, G_{1}}\left(\theta_{1}^{(i)}\right) \\
& =\sum_{\chi \in \hat{D}} \chi \pi_{2}+\sum_{i} i_{G_{2}, G_{1}}\left(\theta_{1}^{(i)}\right) .
\end{aligned}
$$

If $\chi \pi_{2} \leq i_{G_{2}, G_{1}}\left(\theta_{1}^{(i)}\right)$, then $\pi_{1}=\theta_{1}^{(i)}$, which contradicts multiplicity one for $G_{1}$. Thus, for each $\chi \in \hat{D}$, we have $\chi \pi_{2}$ occurs with multiplicity one in $i_{G_{2}, G_{1}} \circ$ $i_{G_{1}, M_{1}}\left(\exp \nu_{1} \otimes \tau_{1}\right)$, hence with multiplicity one in $\bigoplus_{\chi \in \hat{D}} i_{G_{2}, M_{2}}\left(\exp \nu_{2} \otimes \chi \tau_{2}\right)$. Now,

$$
i_{G_{2}, M_{2}}\left(\exp \nu_{2} \otimes \chi \tau_{2}\right) \cong \chi \circ i_{G_{2}, M_{2}}\left(\exp \nu_{2} \otimes \tau_{2}\right)
$$

contains $\chi \pi_{2}$ with multiplicity one. In particular, this means $i_{G_{2}, M_{2}}\left(\exp \nu_{2} \otimes \tau_{2}\right)$ contains $\pi_{2}$ with multiplicity one and no $\chi \pi_{2}$ with $\chi \neq 1$.

Let $\theta$ be an irreducible subquotient of $i_{G_{2}, M_{2}}\left(\exp \nu_{2} \otimes \tau_{2}\right)$. By Lemma 3.6, $C_{2} \cdot \nu_{2} \leq C_{2} \cdot \nu_{\theta}$. Furthermore, if $C_{2} \cdot \nu_{2}=C_{2} \cdot \nu_{\theta}$ (in the ordering), then $\theta$ is a subquotient of $i_{G_{2}, G_{1}}\left(\pi_{1}\right)$, so $\theta \cong \chi \pi_{2}$, for some $\chi$. Since $i_{G_{2}, M_{2}}\left(\exp \nu_{2} \otimes \tau_{2}\right)$ contains no $\chi \pi_{2}$ with $\chi \neq 1$, it follows that $\theta \cong \pi_{2}$ and $\nu_{\theta}=\nu_{2}$.

Theorem 3.4 now follows from Lemmas 3.5 and 3.7, and induction.

Corollary 3.8. In the Grothendieck group, any irreducible representation may be written as a linear combination of standard induced representations, i.e., of representations having the form $i_{G, M}(\exp \nu \otimes \tau)$ with $(P, \nu, \tau)$ Langlands data.

Remark 3.1. As noted in Remark 4.2 in B-J1, the Langlands classification may also be formulated in the quotient setting. In this setting, Theorem 3.4 and Corollary 3.8 hold, with only the change $C \cdot \nu>C \cdot \nu_{\theta}$ in Theorem $3.4,2$, required.

\section{REFERENCES}

[B-J1] D. Ban and C. Jantzen, The Langlands classification for non-connected p-adic groups, Israel Journal of Mathematics, 126 (2001), 239-261. MR 2002i:22018

[B-J2] D. Ban and C. Jantzen, Degenerate principal series for even-orthogonal groups, preprint.

[B-Z] I.N. Bernstein and A.V. Zelevinsky, Induced representations of reductive p-adic groups, I, Annales Scientifiques de l'École Normale Supérieure 10 (1977), 441-472. MR 58:28310

[B-W] A. Borel and N. Wallach, Continuous Cohomology, Discrete Subgroups, and Representations of Reductive Groups - 2nd ed., Mathematical Surveys and Monographs 67 (2000). MR 2000j:22015

[G-K] S. Gelbart and A. Knapp, L-indistinguishability and $R$ groups for the special linear group, Advances in Mathematics 43 (1982), 101-121. MR 83j:22009

[G-H] D. Goldberg and R. Herb, Some results on the admissible representations of non-connected reductive p-adic groups, Annales Scientifiques de l'École Normale Supérieure 30 (1997), 97146. MR 98b:22033

[H] Harish-Chandra, Harmonic analysis on reductive p-adic groups, Proceedings of Symposia in Pure Mathematics 26 (1974), 167-192. MR 49:5238

[J] C. Jantzen, Duality and supports of induced representations for even-orthogonal groups, preprint.

[M] Z. Magyar, Langlands classification for real Lie groups with reductive Lie algebra, Acta Applicandae Mathematicae 37 (1994), 267-309. MR 96i:22035 
[L] R. Langlands, On the classification of irreducible representations of real algebraic groups, Representation Theory and Harmonic Analysis on Semisimple Lie Groups, editors Paul Sally, Jr. and David Vogan, Mathematical Surveys and Monographs, AMS, vol. 31, 1989, pp.101-170. MR 91e:22017

[S] A. Silberger, The Langlands quotient theorem for $p$-adic groups, Mathematische Annalen 236 (1978), 95-104. MR 58:22413

Department of Mathematics, Southern Illinois University, Carbondale, Illinois 62901

E-mail address: dban@math.siu.edu

Department of Mathematics, East Carolina University, Greenville, North Carolina 27858

E-mail address: jantzenc@mail.ecu.edu 